\title{
TNFRSF14 (HVEM) is a novel immune checkpoint for cancer immunotherapy in humanized mice
}

Nicolas Aubert ${ }^{1^{*}}$, Simon, Brunel ${ }^{1^{*}}$, Daniel Olive ${ }^{2}$ and Gilles Marodon ${ }^{1}$

1 Sorbonne Universités, Inserm, CNRS, Centre d'immunologie et maladies infectieuses-Paris, Cimi-Paris, Paris 75013, France

2 Institut Paoli-Calmettes, Aix-Marseille Université, Inserm, CNRS, CRCM, Tumor Immunity Team, IBISA Immunomonitoring platform, Marseille, France (daniel.olive@inserm.fr)

* Equal contributions (nicolas.aubert@inserm.fr, simonbrunel@live.fr)

Running title: HVEM is a novel target for cancer immunotheraoy

Corresponding author: Gilles Marodon, gilles.marodon@inserm.fr, Sorbonne Université, Inserm, CNRS, Centre d'immunologie et maladies infectieuses-Paris (CIMI-PARIS), Paris 75013, France

Categorie: Immune Cell Therapies and Immune Cell Engineering

Word count: 4928

\section{Abstract}

2 Background: TNFRSF14 (herpes virus entry mediator (HVEM) delivers a negative signal to T cells through

3 the B and T Lymphocyte Attenuator (BTLA) molecule and has been associated with a worse prognosis in

4 numerous malignancies. A formal demonstration that the HVEM/BTLA axis can be targeted for cancer

5 immunotherapy is however still lacking.

6 Methods: We used immunodeficient NOD.SCID.gc-null mice reconstituted with human PBMC and grafted

7 with human tumor cell lines subcutaneously. Tumor growth was compared using linear and non linear

8 regression statistical modeling. The phenotype of tumor-infiltrating leukocytes was determined by flow

9 cytometry. Statistical testing between groups was performed by a non-parametric $t$ test. Quantification of

10 mRNA in the tumor was performed using NanoString pre-designed panels. Bioinformatics analyses were 
10 performed using Metascape, Gene Set Enrichment Analysis and Ingenuity Pathways Analysis with embedded

11 statistical testing.

12 Results: We showed that a murine monoclonal antibody to human HVEM significantly impacted the growth

13 of various HVEM-positive cancer cell lines in humanized NSG mice. Using CRISPR/cas9 mediated deletion

14 of HVEM, we showed that HVEM expression by the tumor was necessary and sufficient to observe the

15 therapeutic effect. Tumor cell killing by the mAb was dependent on innate immune cells still present in NSG

16 mice, as indicated by in vivo and in vitro assays. Mechanistically, tumor control by human $\mathrm{T}$ cells by the

$17 \mathrm{mAb}$ was dependent on CD8 $\mathrm{T}$ cells and was associated with an increase in the proliferation and number of

18 tumor-infiltrating leukocytes. Accordingly, the expression of genes belonging to $\mathrm{T}$ cell activation pathways,

19 such as JAK/STAT and NFKB were enriched in anti-HVEM-treated mice, whereas genes associated with

20 immuno-suppressive pathways were decreased. Finally, we developed a simple in vivo assay to directly

21 demonstrate that HVEM/BTLA is an immune checkpoint for T-cell mediated tumor control.

22 Conclusions: Our results show that targeting HVEM is a promising strategy for cancer immunotherapy.

\section{Keywords}

immune checkpoint; HVEM; BTLA; monoclonal antibody; cancer immunotherapy; humanized mice

\section{Introduction}

24 Immune escape by tumor is now considered a hallmark of cancer [1]. Many immune mechanisms are

25 involved to explain the loss of tumor control, including defective MHC function and expression, recruitment

26 of suppressive immune cells, and expression of co-inhibitory receptors such as PD-L1 [2]. In the last few

27 years, targeting co-inhibitory molecules with antibodies has shown impressive results in tumor regression

28 and overall survival, leading to the approval of anti-CTLA-4, anti-PD-1 and anti-PD-L1 in numerous cancers

29 [3]. However, the success of immune checkpoint inhibitors (ICI) is still partial and many patients fail to

30 respond. Limited tumor infiltrate (cold tumors) or low expression of the targeted molecule may explain the

31 relative inefficiency of ICI $[4,5]$. To overcome these limitations, it is necessary to explore other pathways

32 that might be involved in immune escape and that could complement actual therapies. 
33 Recently, a new co-inhibitory pair has been highlighted in anti-tumor immune response: HVEM (Herpes

34 Virus Entry Mediator, TNFRSF14) and BTLA (B and T lymphocyte attenuator) [6]. These two molecules can

35 be expressed by many immune cells, including T-cells, in which signaling through BTLA is associated with

36 inhibition of their activation [7,8]. Additionally, the HVEM network includes many additional partners, such

37 as LIGHT, Herpes Simplex Virus-1 (HSV-1) glycoprotein D (gD), lymphotoxin $\alpha$ (LT $\alpha$ ) or CD160 [6]. Like

38 BTLA, binding of HVEM to CD160 on T-cells is associated with an inhibition of their activation [9]. In

39 contrast, LIGHT is clearly a T-cell activator since transgenic expression of LIGHT in T cells leads to massive

40 activation, especially in mucosal tissues [10]. On the other hand, stimulation of HVEM expressed by T-cells

41 by any of its ligands is associated with proliferation, survival and production of inflammatory cytokines,

42 such as IL-2 and IFN- $\gamma[9,11]$. Several clinical studies have shown that HVEM expression is upregulated in

43 many types of cancers including colorectal cancers [12], melanomas [13], esophageal carcinomas [14],

44 gastric cancers [15], hepatocarcinomas [16], breast cancers [17], lymphomas [18] or prostate cancer [19]. In

45 these studies, high levels of HVEM expression by tumors were associated with a worse prognosis and lower

46 survival. Moreover, HVEM expression by tumors was also associated with a reduction in the numbers of

47 tumor-infiltrating leukocytes (TIL) $[12,14,16]$.

48 Few studies have considered targeting the HVEM network to affect tumor growth. In fact, various strategies

49 to inhibit HVEM expression or function lead to increased T cell proliferation and function in syngeneic

50 tumor mouse models [14,20,21]. However, to our knowledge, no study to date has assessed the possibility to

51 use a monoclonal antibody (mAb) to HVEM to favor the anti-tumor immune response in a humanized

52 context in vivo. Herein, we investigated the therapeutic potential of a murine antibody targeting human

53 HVEM in humanized mice grafted with various human tumor cell lines. To generate humanized mice, we

54 used immuno-compromised NOD.SCID.yc ${ }^{\text {null }}$ (NSG) mice, which are deprived of murine T-, B- and NK-

55 cells but that retain functionally immature macrophages and granulocytes [22]. We reconstituted these mice

56 with human PBMC, allowing the effect of blocking HVEM to be studied on both tumors, murine myeloid

57 cells and human T-cells.

\section{Methods}


Preparation of human peripheral mononuclear cells

60 Human peripheral blood mononuclear cells were isolated on a density gradient (Biocoll). Cells were washed

61 in PBS 3\% FCS and diluted at the appropriate concentration in 1X PBS before injection into mice.

\section{Humanized mice tumor model}

63 All animals used were NSG mice (stock $\neq 005557$ ) purchased from the Jackson Laboratory (USA). To assess

64 therapeutic activity, 8-20-week-old NSG mice (males and females) were injected subcutaneously with $2.10^{6}$

65 tumor cells. One week later, mice were irradiated (2 Gy) and grafted the same day with $2.10^{6}$ huPBMC by

66 retro orbital injection. Four to 5 days after transplantation, the anti-huHVEM antibody or isotype control was

67 injected intra-peritoneally at $2 \mathrm{mg} / \mathrm{kg}$. General state, body weight and survival of mice were monitored every

68 3-4 days to evaluate Graft-vs-Host-Disease (GVHD) progression. Mice were euthanized when exhibiting

69 signs of GVHD, such as hunched back, ruffled fur, and reduced mobility. For CD8 depletion, mice were

70 injected intra-peritoneally with $10 \mathrm{mg} / \mathrm{kg}$ of the anti-CD8 MT807R1( Rhesus recombinant IgG1 provided by

71 the Nonhuman Primate Reagent Resource [23]) or the isotype control (clone DSPR1) the day following

72 humanization, as previously described [24].

\section{Antibodies}

74 The clone 18.10 has been described previously [25]. Briefly, 18.10 is a murine IgG1 anti-human HVEM

$75 \mathrm{mAb}$ and was produced as ascites and purified by protein A binding and elution with the Affi-gel Protein A

76 MAPS II Kit (Bio-rad). The mouse IgG1 isotype control (clone MOPC-21 clone), the rat IgG2b anti-Gr1

77 (clone RB6-8C5) and the isotype control rat IgG2b (clone LTF-2) were purchased from Bio X Cell (West

78 Lebanon, NH, USA).

79 Cell lines

80 PC3 (non-hormono-dependent human prostate cancer cells), Gerlach (human melanoma cells), MDA-MB-

81231 (breast cancer cells), DU145 (prostate cancer cells) were grown in high glucose DMEM media

82 supplemented with 10\% FCS, L-glutamine and antibiotics (Penicillin/Streptomycin). PC3 and MDA-MB-

83231 were genetically authenticated before the initiation of the experiments (Eurofins). All cells were

84 confirmed to be free of mycoplasmas before injection into mice by the MycoAlert detection kit (Lonza).

85 Tumor growth was monitored using an electronic caliper and volumes were determined using the following 
86 formula: [(length*width²)/2]. The PC3-GFP cell line was generated in the laboratory by lentiviral

87 transduction (details available on request).

88 Generation of HVEM deficient PC3 clone using CRISPR-Cas9 technology

89 50,000 PC3 cells were seeded in a 24-well plate. Twenty-four hours later, cells were incubated with sgRNA

90 complementary to exon 3 of HVEM (GCCAUUGAGGUGGGCAAUGU + Scaffold, TrueGuide Synthtetic

91 guide RNAs, Invitrogen ${ }^{\mathrm{TM}}$ ), Cas9 nuclease (TrueCut ${ }^{\mathrm{TM}}$ Cas9 Protein v2, Invitrogen ${ }^{\mathrm{TM}}$ ) and lipofectamine

92 (Lipofectamine ${ }^{\mathrm{TM}}$ CRISPRMAX ${ }^{\mathrm{TM}}$ Cas9 Transfection Reagent, Invitrogen ${ }^{\mathrm{TM}}$ ) according to manufacturer

93 instructions (TrueCut Cas9 protein v2 (27/09/2017)). After three days, efficiency was evaluated with

94 GeneArt Genomic Cleavage Detection Kit (Invitrogen ${ }^{\mathrm{TM}}$ ) according to the manufacturer instructions. For

95 this assay, DNA was amplified with the following primers: TGCGAAGTTCCCACTCTCTG (Forward) and

96 GGATAAGGGTCAGTCGCCAA (Reverse). Cells were cloned by limiting dilution in 96-well plates. Clones

97 were screened for HVEM expression by flow cytometry using anti-HVEM (clone 94801, BD) and were

98 considered as negative if HVEM expression was undetectable for at least 3 subsequent measurements.

99 In vitro assays

100 PC3 cells were seeded in 96-wells plate at 7000 cells/well in RPMI medium. Cells were treated by the anti

101 HVEM antibody or its isotype control MOPC21 coated at $10 \mu \mathrm{g} / \mathrm{ml}$. Cell death was evaluated by flow

102 cytometry after 16 hours of incubation $\left(37^{\circ} \mathrm{C}, 5 \% \mathrm{CO}\right)$ by $7-\mathrm{AAD}$ staining. Macrophages from NSG mice

103 were obtained by peritoneal wash. The target to effector ratio was 1:5 for apoptosis monitoring. For live cell

104 imaging, apoptosis of the PC3 GFP cell line was assessed using the annexin V red (cat $n^{\circ} 4641$, Sartorius).

105 Culture was monitored every hour during 16 hours by Incucyte and overlapping of GFP (green) and

106 apoptosis staining (red) was quantified and reported as number of apoptotic cells/well.

107 Phenotypic analysis by flow cytometry

108 Tumors were digested with $0.84 \mathrm{mg} / \mathrm{mL}$ of collagenase IV and $10 \mu \mathrm{g} / \mathrm{mL}$ DNAse I (Sigma Aldrich) for $40 \mathrm{~min}$

109 at $37^{\circ} \mathrm{C}$ with an intermediate flushing of the tissue. Cells were passed through a $100 \mu \mathrm{m}$-cell strainer and

110 suspended in PBS 3\% FCS. To eliminate dead cells and debris, tumor cell suspensions were isolated on a

111 Biocoll gradient. Rings were collected, washed, and cell pellets were suspended in PBS 3\% FCS before

112 counting on LUNA ${ }^{\mathrm{TM}}$ Automated Cell counter (Logos Biosystems). Subsequently, up to $2.10^{6}$ live cells were

113 stained with viability dye (eF506, Fixable Viability Dye, ThermoFisher) for $12 \mathrm{~min}$. at $4^{\circ} \mathrm{C}$, Fc receptor were 
114 blocked with human FcR Blocking Reagent (120-000-442, Miltenyi Biotec) and anti-CD16/32 (clone 2.4G2)

115 for $10 \mathrm{~min}$. The followings antibodies were added for $35 \mathrm{~min}$. at $4^{\circ} \mathrm{C}$ : hCD45-BUV805 (HI30, BD), hCD3116 PECyn7 (SK7, BD), hCD4-PerCP (RPA-T4, Biolegend), hCD8-APC-H7 (SK1, BD), hKi67-AF700 (B56, 117 BD), hCD270-BV421 (cw10, BD), and mCD45-BUV395 (30-F11, BD) hGranzymeB-APC (GB11, 118 eBioscience), hPerforin-PE (B-D48, Biolegend) and mCD45-BUV395 (30-F11, BD). For intracellular 119 staining, Foxp3/Transcription Factor Staining (eBioscience) or Cytofix/Cytoperm (BD) buffer sets were 120 used. Cells were washed with 1X PBS before acquisition on an X20 cytometer (Becton Dickinson (BD), San 121 Jose, CA). The absolute count of different populations was determined by adding $50 \mu \mathrm{L}$ of Cell Counting 122 Beads (Bangs Laboratories) before acquisition. Data were analyzed using FlowJo software (TreeStar, 123 Ashland, OR, USA).

\section{NanoString nCounter expression assay}

125 For Nanostring ${ }^{\circledR}$ experiment, 14 to 15 weeks-old NSG mice were humanized and treated with anti-HVEM 126 or isotype. Day 28 post humanization, tumors were harvested and TIL were isolated as described above. To 127 maximize mRNA recovery, TIL were pooled by treatment groups (4 mice in the anti-HVEM group and 5 in 128 the isotype control group). Then, cells were stained with viability dye (eF506) and anti hCD45-APC (HI30, Biolegend). Live hCD45 ${ }^{+}$cells were sorted using Aria II cell sorter. After centrifugation, cells were suspended in RLT buffer (Qiagen ${ }^{\circledR}$ ) before freezing at $-80^{\circ} \mathrm{C}$ until analysis. Data were normalized through

131 the use of NanoString's intrinsic negative and positive controls according to the normalization approach of 132 the nSolver analysis software (Nanostring). For the analysis, 287 genes with raw count higher than 55 and an 133 absolute fold-change of at least 20\% were retained. Enrichment analysis was performed with Metascape 134 [26], the GSEA desktop application [27] and Ingenuity Pathway Analysis (IPA) (Qiagen). For Metascape analysis, genes up or down regulated were analyzed separately whereas all genes were included in the GSEA or IPA analyses. For GSEA analysis, enrichment was performed using the Hallmark v7.2 or the Canonical Pathways v7.2 gene sets from the Broad Institute. With that workflow, a False Discovery Rate (FDR) or a

138 Family Wise Error Rate (FWER) less than 0.25 is deemed "significant”.

139 Statistical analysis

140 All statistical tests were performed with Prism software (Graph Pad Inc, La Jolla, CA, USA). To compare 141 ranks between two groups, the p-value was calculated with a non-parametric two-tailed Mann-Whitney t-test. 
142 Statistical modeling of tumor growth was performed by linear and non-linear regression using the

143 exponential growth model. When necessary, the p-values of these tests are indicated on the figure panels.

144 Statistical power of the analyses (alpha) was arbitrarily set at 0.05 . No test was performed $a$ priori to

145 adequate the number of samples with statistical power.

\section{Results}

\section{Targeting HVEM with a mAb improves tumor control of HVEM+ cell lines in humanized mice}

148 We first determined whether HVEM could be targeted for therapy by the anti-HVEM 18.10 monoclonal 149 antibody. For that, we implanted various tumor cell lines in NSG mice and grafted human PBMCs few days 150 after. No differences in tumor growth were observed with mice grafted with the prostate cancer cell line 151 DU145 or with the triple-negative breast cancer cell line MDA-MB-231, which did not express HVEM 152 (Figure 1A-B). In contrast, a significant reduction of tumor growth was observed in mice grafted with the 153 HVEM-positive patient-derived melanoma cell line Gerlach and the PC3 prostate cancer cell line (Figure 154 1C-D). To rule out that the effect of the mAb on tumor growth was due to other differences than HVEM 155 expression, we generated an HVEM-deficient PC3 cell line (clone 1B11) using CRISPR-Cas9 156 ribonucleoprotein (RNP) transfection (Figure 1E). The treatment with the mAb was completely inefficient on 157 the 1B11 cell line in humanized mice (Figure 1E). Of note, the knock-down of HVEM impacted tumor 158 growth to the same extent than the mAb on HVEM ${ }^{+}$PC3 cells (Figure 1D-E). Thus, HVEM expression on 159 the tumor was mandatory for the therapeutic efficacy of the mAb. 
A

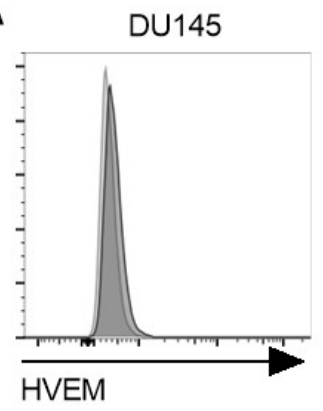

B

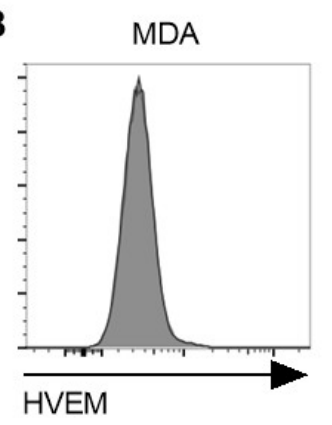

C

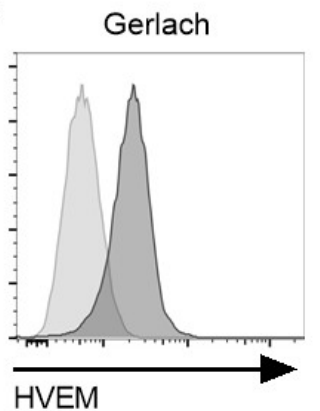

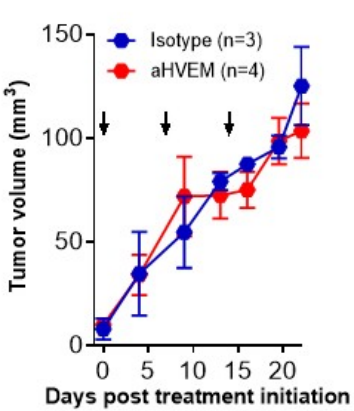

D

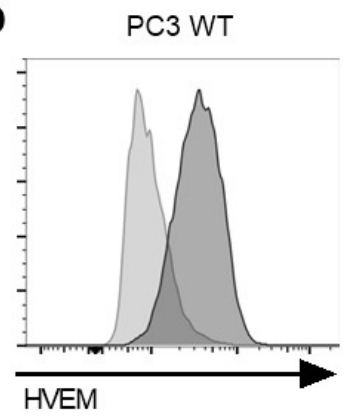

E
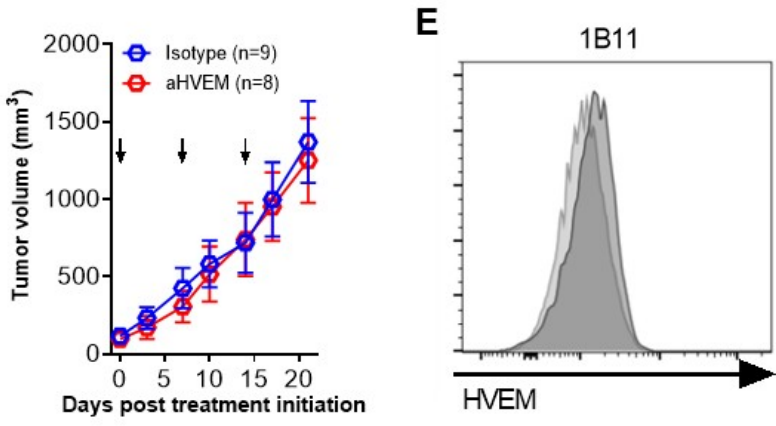
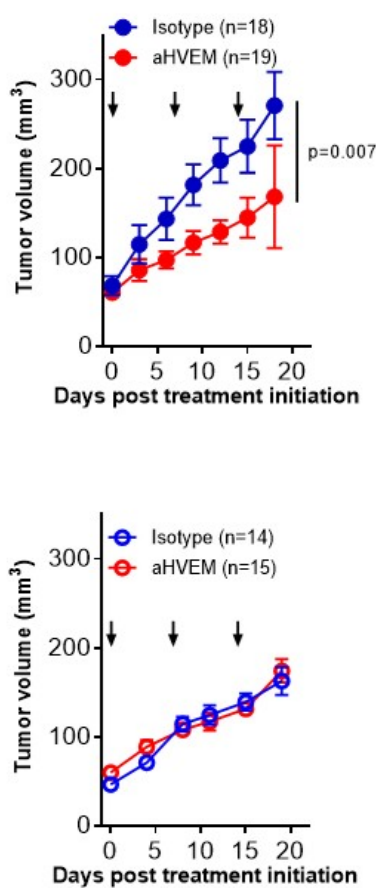

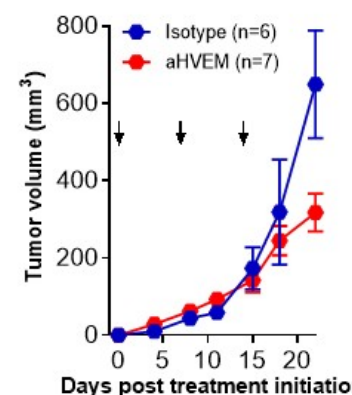

Days post treatment initiation

Figure 1: Targeting HVEM with a mAb improves tumor control of HVEM+ cell lines in humanized mice. HVEM expression and tumor growth of the prostate cancer cell line DU145 (A), the breast cancer cell line MDA-MB-231 (MDA) (B), the patient derived melanoma Gerlach (C), the prostate cancer cell line PC3 (D) and the HVEM-deficient PC3 clone 1B11. HVEM expression was determined by flow cytometry with the anti-HVEM mAb (clone 18.10) and a secondary antibody. Curves represent the mean tumor volume ( \pm SEM) from one experiment with DU145 and at least two for the others. Numbers of mice at the beginning of the experiments are indicated in brackets. Arrows indicate the time of the injections. The p value on the graphs indicate the probability that the slopes are equal using a linear regression model.

NSG myeloid cells are able to kill PC3 cells in presence of the anti-HVEM antibody

169 We next evaluated whether the mAb would mediate direct killing of tumor cells since HVEM has been

170 linked to pro apoptotic signaling [28]. However, the anti-HVEM mAb was unable to induce tumor cell death

171 in vitro (Figure 2A). In contrast, a significant reduction in tumor growth after mAb treatment was observed 
172 for the parental PC3 cell line in non-humanized NSG mice (Figure 2B). Because NSG mice are on a NOD

173 genetic background which is deficient for complement activity [22], we surmised that innate immunity of

174 NSG mice might be involved in the activity of the mAb. Indeed, depletion of monocytes and neutrophils

175 with an anti-Gr1 mAb completely reverted the effect of the treatment, but a high mortality of NSG mice was

176 observed (Figure S1). We thus co cultured PC3 cells with macrophages obtained from peritoneal lavage of

177 NSG mice (Figure S2). Using live imaging, we observed a progressive increased proportion of apoptotic

178 cells in presence of the anti-HVEM mAb (Figure 2C). Furthermore, video microscopy of the co-cultures

179 revealed that tumor cells were killed by a cell-contact dependent mechanism with no evidence for 180 engulfment of tumor cells (Video S1). Thus, innate immunity of NSG mice is not passive during treatment

181 and may participate in tumor killing following treatment with the mAb.

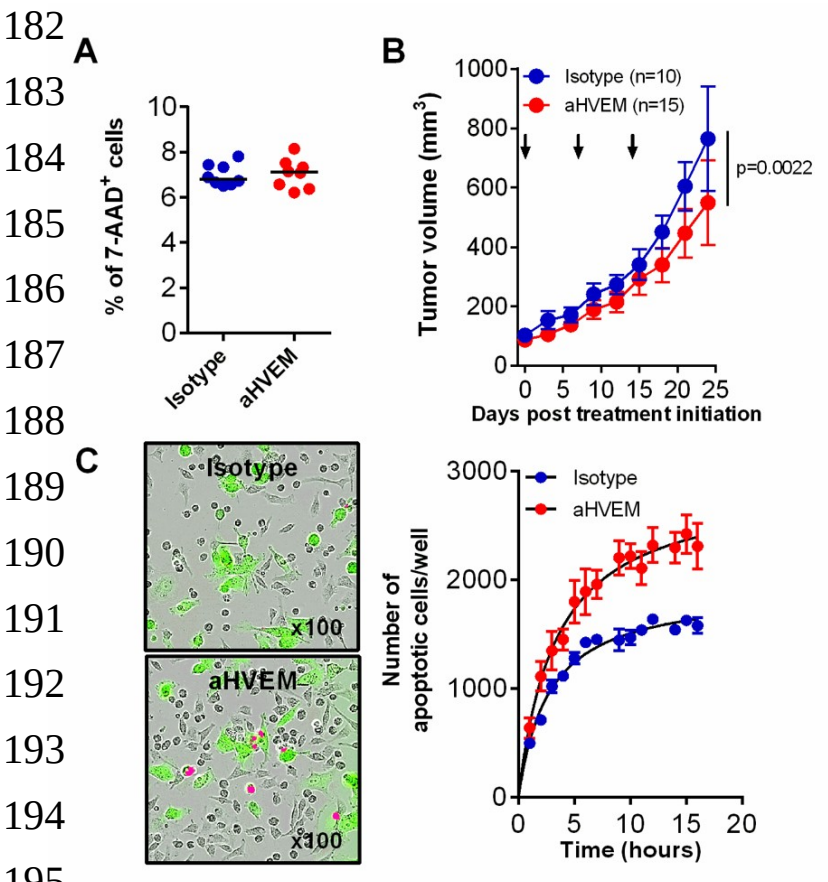

Figure 2: NSG myeloid cells are able to kill wild-type PC3 cells in presence of the anti-HVEM antibody. (A) Frequencies of 7AAD+ cells on the parental cell line PC3 in culture with anti-HVEM or isotype control mAb were determined by flow cytometry. (B) Tumor growth of the parental PC3 cell line in non-humanized NSG mice treated with the anti-HVEM or Isotype control mAb. Data are cumulative of 3 independent experiments. (C) GFPexpressing wild-type PC3 cells were co-cultured with NSG peritoneal macrophages and an apoptosis staining reagent. Magnification is indicated. (D) Overlap of GFP (green) and apoptosis staining (red) was quantified and reported as number of apoptotic cells/well \pm SEM of technical replicates. Depicted are the results from one experiment.

\section{Treatment with the anti-HVEM mAb 18.10 results in an increase in TIL number and proliferation}

197 To dig further into the mode of action of the mAb, we determined the relative frequencies of murine and

198 human $\mathrm{CD}_{4} 5^{+}$cells in the tumor by flow cytometry. Among all CD45+ cells, murine cells were very rare 199 compared to human cells. Human cells represented more than $90 \%$ of all CD45+ cells, in which CD3+CD4+ 200 and CD8+ represented more than 95\% (Figure 3A-C), showing that the tumor was mostly infiltrated by 
201 human T-cells. These proportions were not changed by the treatment. In contrast, we observed an increase in

202 CD4 T-cells numbers and a similar tendency for CD8 T-cells in the anti-HVEM-treated group (Figure 3D).

203 Additionally, frequencies of cells expressing the proliferation marker Ki67 were significantly elevated in 204 both CD4 and CD8 T-cells (Figures 3E).

A
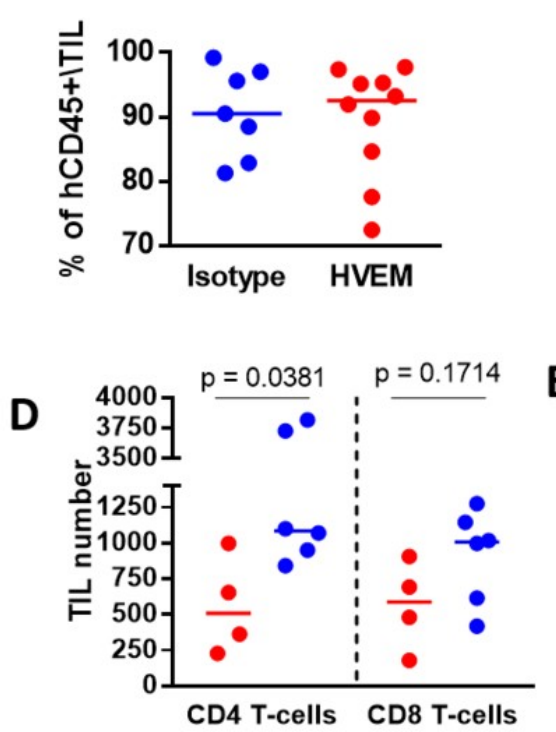

B

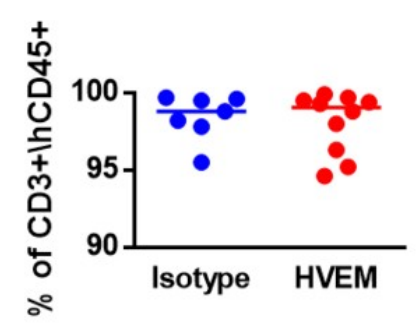

E

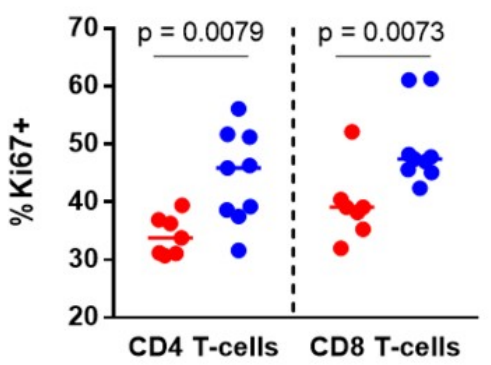

205 Figure 3: Treatment with the anti-HVEM mAb 18.10 results in an increase in TIL number and proliferation. Frequencies of human CD45+ cells among all CD45+ cells (A), of human CD3+ among human CD45+ cells (B) and of CD4 and CD8 T cells among human CD3+ cells (C ) in the PC3 tumor were determined by flow cytometry. (D) Total number of $\mathrm{CD}^{+}$and $\mathrm{CD}^{+} \mathrm{T}$-cells in PC3 tumors from one representative experiment out of 2. (E) Frequencies of Ki67-expressing cells among CD4 ${ }^{+}$and $\mathrm{CD}^{+} \mathrm{T}$-cells in the tumor. Data are cumulative of two independent experiments performed at D21 post-humanization.

211 Each dot is a mouse. The p values on the graphs indicate the probability that the median values were equals

212 using the Mann-Whitney non parametric t-test.

\section{Tumor control is dependent on $\mathbf{C D 8}^{+} \mathbf{T}$ cells}

214 To determine the contribution of CD8+ T cells to tumor control, we compared tumor growth in anti-HVEM-

215 treated mice in mice depleted of their CD8+ T cells (Figure 4). Depletion of CD8 T-cells was clearly visible

216 at the end of the experiment in the tumor (Figure 4A), showing that the depleting mAb had a long-lasting

217 effect. Interestingly, depletion of CD8+ T cells before the initiation of the treatment reverted the effect of the

218 anti-HVEM mAb on tumor growth (Figure 4B), showing that CD8 T cells were crucial for the therapeutic

219 efficacy of the mAb. However, Granzyme B (GZMB) and Perforine 1 (PRF1) expression levels were not 
220 elevated in $\mathrm{CD}^{+} \mathrm{T}$ cells of the tumor of treated mice (Figure 4C-D), indicating that tumor control was

221 dependent on CD8 T cell numbers rather than function.

A

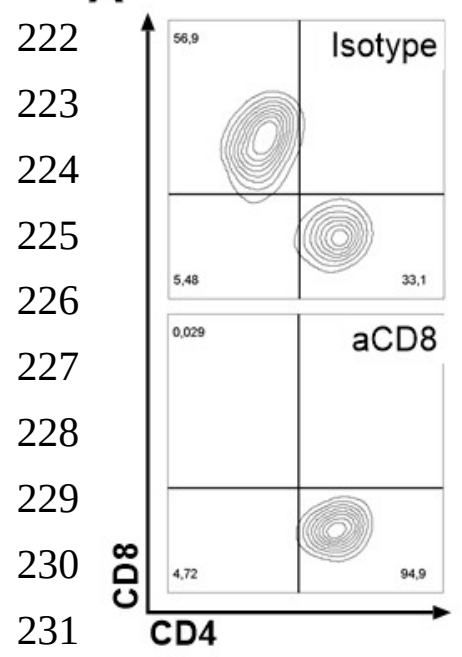

${ }^{232} \mathbf{C}$

233

234

235

236
B
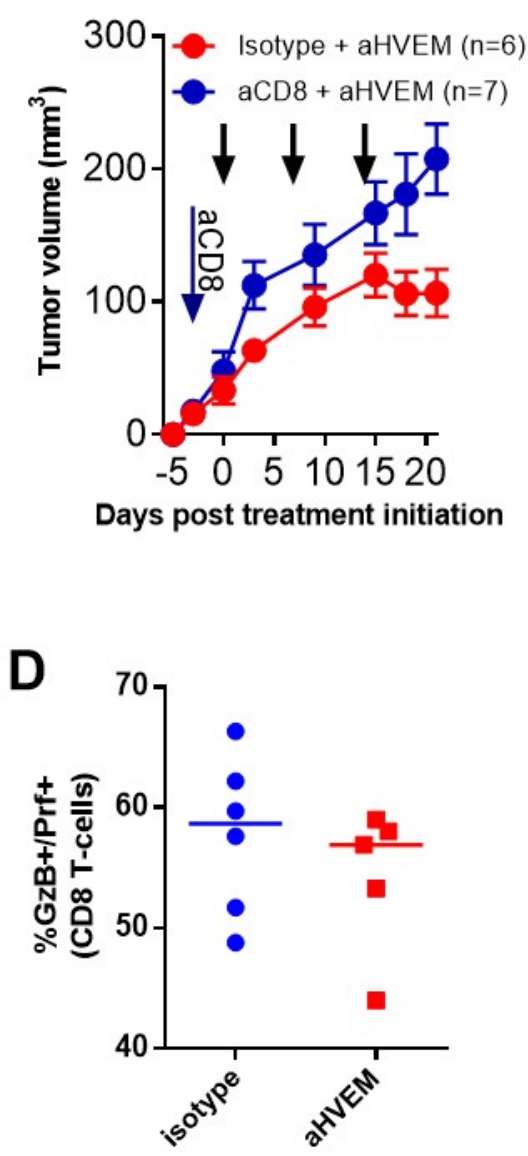

Figure 4: Tumor control by the mAb is dependent on $\mathrm{CD8}^{+} \quad \mathbf{T}$ cells (A) Representative CD4/CD8 staining on human CD45+CD3+ T-cells in the tumor at the end of the experiment in a CD8-depleted (aCD8) or an isotype control treated mouse. (B) Growth of the PC3 cell line in humanized mice treated with anti-HVEM mAb and depleted or not of their CD8 T cells. CD8 Tcells were depleted on the day following humanization (blue arrow). Curves are the mean tumor volume $( \pm S E M)$ in the indicated number of mice. Black arrows indicate the time of anti-HVEM mAb injection. Data are cumulative of two independent experiments.

\section{Treatment with the anti-HVEM mAb does not increase GVHD nor number or proliferation of human}

\section{T cells}

239 One possibility to explain these observations would be that the mAb behave as an agonist, directly activating

240 human T cells in vivo, leading to better tumor control. Indeed, human T and B cells did express HVEM

241 before injection into mice (Figure 5A). However, we observed similar weight loss and mortality in anti-

242 HVEM or isotype control treated mice (Figure 5B-C), showing that GVHD induced by PBMC in NSG mice

243 was not exacerbated by the treatment. Furthermore, the number and the proliferation status of human $\mathrm{T}$ cells

244 in the spleens of treated animals were the same (Figure 5D-E). Our results show that anti-HVEM therapy in 
245 humanized mice reduced the growth of $\mathrm{HVEM}^{+}$tumors by a mechanism that was independent of any agonist

246 effect of the mAb.

247

248

249

250

251

252

253

254

255

256

257
A
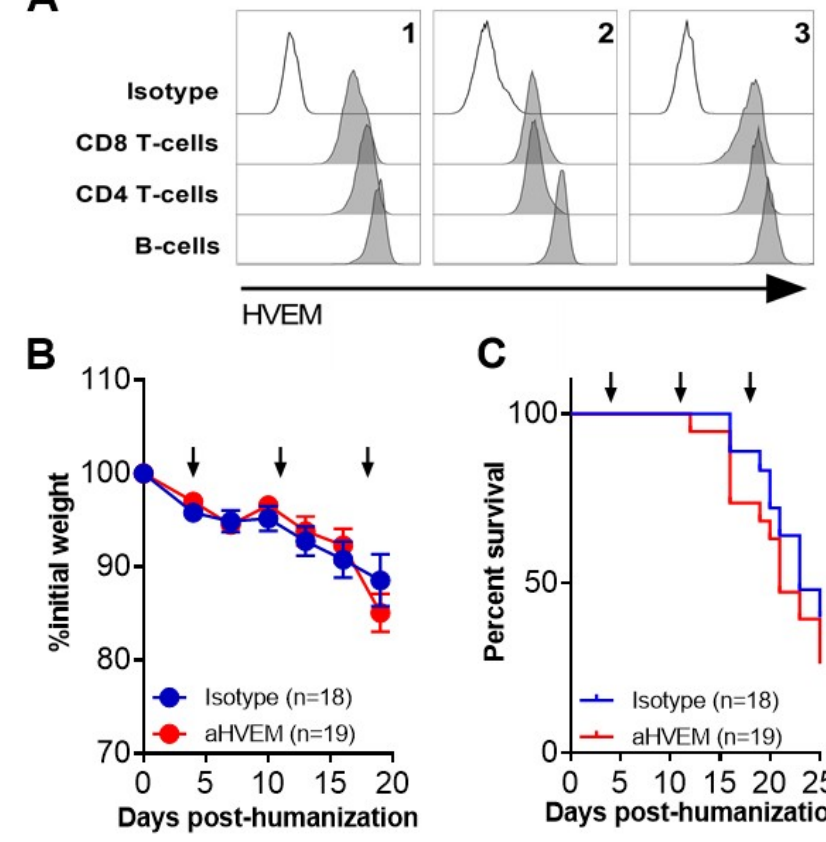

D

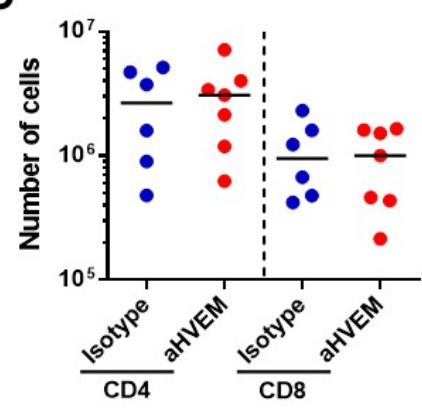

C

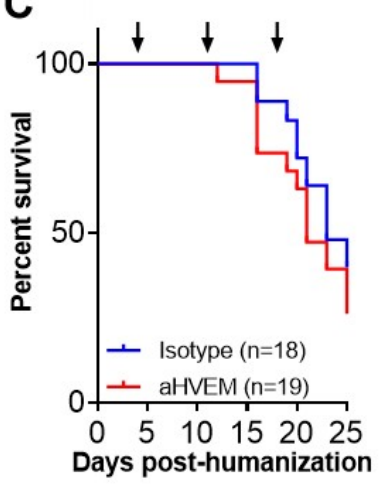

E

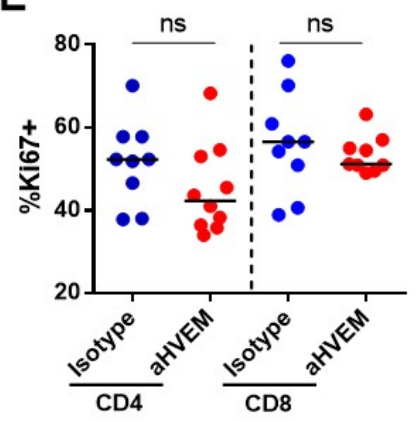

Figure 5: Treatment with the anti-HVEM mAb does not increase GVHD nor numbers or proliferation of human T cells. (A) HVEM expression in the indicated subsets was determined by flow cytometry on 3 different PBMC donors. Percentages of initial weight (B) and survival (C) of NSG mice following treatment by the anti-HVEM mAb or an isotype control are shown. Numbers (D) and frequencies of Ki67+ cells (E) in the indicated subsets.

mRNA enrichment analysis showed increased activation and decreased immunosuppression in TIL of

260 In order to better characterize the anti-tumor immune response following mAb treatment, we established a

261 list of differentially expressed genes (DEG) in sorted hCD45 $5^{+}$TIL using the Nanostring Cancer Immune panel. Among the 730 genes included in the panel, 145 were up-regulated (log2FC>0.26) and 142 were down-regulated (log2FC<-0.3) in TIL from HVEM-treated mice (Figure S3). Of note, GZMB and PRF1 were among the genes with the highest levels of expression but the difference between the groups was weak, 
enriched by the treatment, such as IL1A, IL7, IL22, EBI3, CSF2, and LTA, a ligand of HVEM. Likewise, the chemokines genes CCL5, CCL4, CCL1, CCL20 and others were enriched by the treatment. Finally, several members of the TNF super family were also enriched, such as TNFSF14 (LIGHT), another ligand of HVEM (Figure S3). Accordingly, unsupervised enrichment analysis revealed that up-regulated genes of the antiHVEM group were enriched in members of several signatures related to interleukins/cytokines, and to

271 activation signaling pathways, including the JAK-STAT, TNF-dependent NFkB and MAPK cascades (Figure 272 6A). Accordingly, NFKB1 and RELA were putative regulators of many genes of the DEG signature (Figure 273 S4A). In addition, Gene Set Enrichment Analysis (GSEA) identified the "JAK-STAT signaling pathway" 274 signature as significantly and positively enriched in TIL of HVEM-treated mice (Figure 6B).

275 On the other hand, some genes belonging to immuno-suppressive pathways were clearly down-regulated in 276 HVEM-treated TIL such as ENTPD1 (CD39), IL10 and the co-inhibitory receptors BTLA, TIGIT, LAG3 277 and HAVCR2 (TIM3), as well as the “don’t eat me” receptor CD47 (Figure S3). Other cytokines/chemokines 278 were also negatively affected by the treatment, such as IL21, IL13, CXCL13, TNFSF10 (TRAIL) or 279 TNFSF113B (BAFF). Enrichment analysis of the genes down regulated in the anti-HVEM group using the 280 DisGeNET database showed that the "Immunosuppression” signature was highly enriched in this gene set 281 (Figure 6C). In addition, GSEA showed that genes belonging to the "immunoregulatory interactions between 282 a lymphoid and a non lymphoid cell” signature was significantly depressed in the DEG signature (Figure 283 6D). In addition, IPA identified several “adhesion and/or binding of lymphocytes/leukocytes” signatures 284 dependent on CSF2 and IL4 as the most significant biological functions associated with the DEG signature 285 (Figure S4B). Overall, anti-HVEM treatment was associated with profound modifications of TIL, with an 286 increased expression of genes belonging to activation and proliferation signaling pathways and a decreased expression of genes signing an exhausted phenotype. 
A

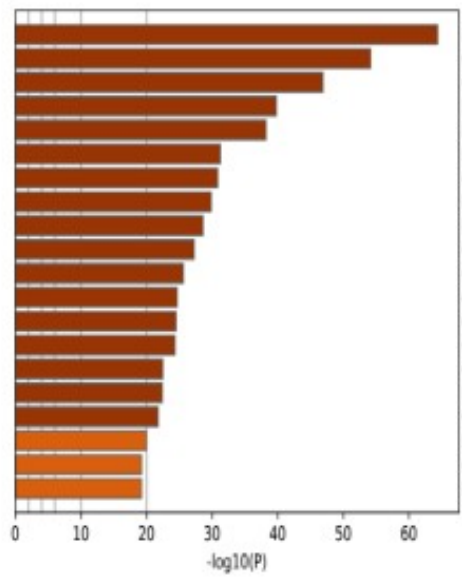

C

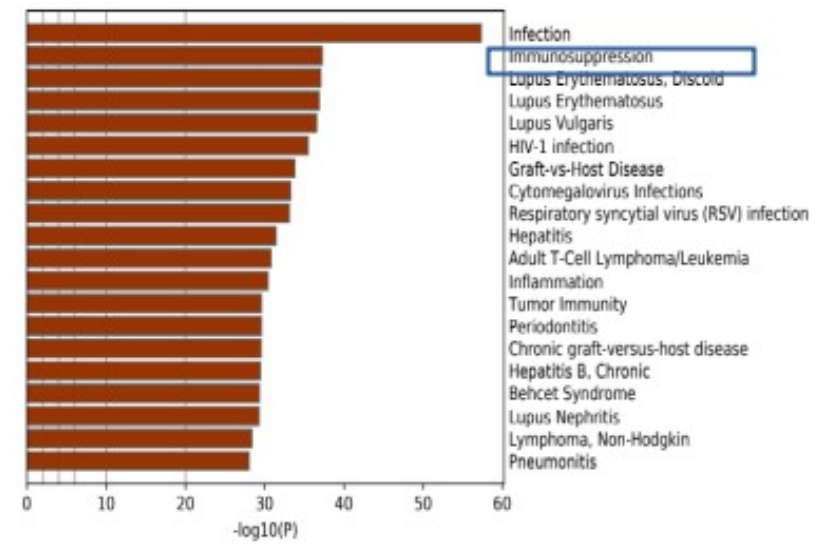

B

R-1SA-409147: Signaling by hterleukins GO:0046649: lymphocyte activation GO:0001819: positive regulation of cytokine production hso05161: Hepastitis B hsa 04060: Cy tokine-cytokine recestor interaction 6004630 : ak. STAT signaling pathwe;

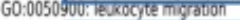

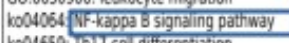
k004659: Thit ceel differentistion G0:0002250: adastive immune response GO:0034612 resporse to tumtor necrosis factor

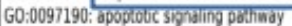
G0:0009615: resporse to virus CO.0002337. 60:0002237: response to molecule of bacterial origin G.0043410 positive regulaztion of MACK cascade GO:0043068: positive regulestion of programmed cell death ho04650: Natural killer cell mediated cytotoxicity 60:0002366: levkocyte activation involved in immune response ko04640: Hematcocietic cell lineage (2)

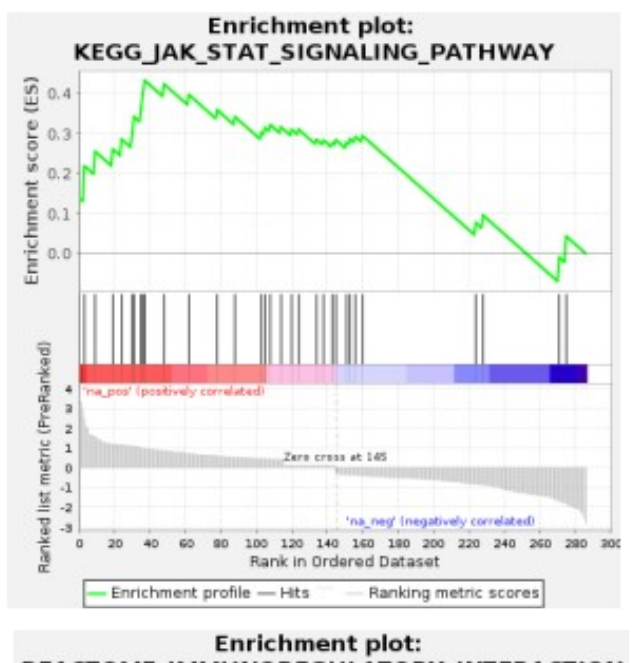

D

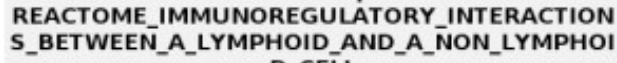
D_CELL

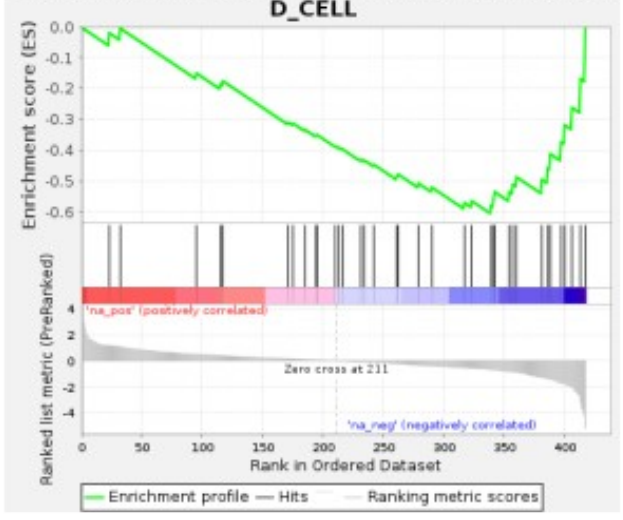

Figure 6: mRNA enrichment analysis show increased activation and decreased immunosuppression in TIL of anti-HVEM treated mice. Quantification of mRNA in CD45+ TIL of anti-HVEM or isotype-treated mice was performed with the Cancer Immune Nanostring panel in PC3-bearing humanized mice. (A) The

291 first 20 terms significantly enriched in up regulated genes of CD45+ TIL of HVEM-treated mice are shown.

292 (B) GSEA of the up-regulated genes identified the "JAK-STAT signaling pathway" signature as significantly 293 enriched (p.val=0,01, q.val=0,26 (FDR), p.val=0,35 (FWER) in CD45+ TIL. (C) The first 20 terms 294 significantly enriched in the genes down regulated by the anti-HVEM treatment are shown according to the 295 DisGeNET database. (D) The "Immunoregulatory interaction between a lymphoid and a non lymphoid cell" 296 signature was significantly enriched (p.val=0,002, q.val=0,148 (FDR), p.val=0,265 (FWER) in genes down 297 modulated by the treatment. 
299 To formally demonstrate that HVEM expression by the tumor was indeed an immune checkpoint, we devised

300 a simple in vivo assay. We implanted the HVEM-positive or the HVEM-negative PC3 cells in NSG mice, and

301 compared tumor growth with or without human PBMCs (Figure 7). Both cell lines grew equally well in non-

302 humanized NSG mice (Figure 7A), showing that HVEM-deficiency did not impacted in vivo tumor

303 development per se. In contrast, tumor growth of the 1B11 clone was reduced compared to the parental PC3

304 cell line in humanized mice (Figure 7B), directly showing that the lack of HVEM improved tumor control by

305 human T-cells.

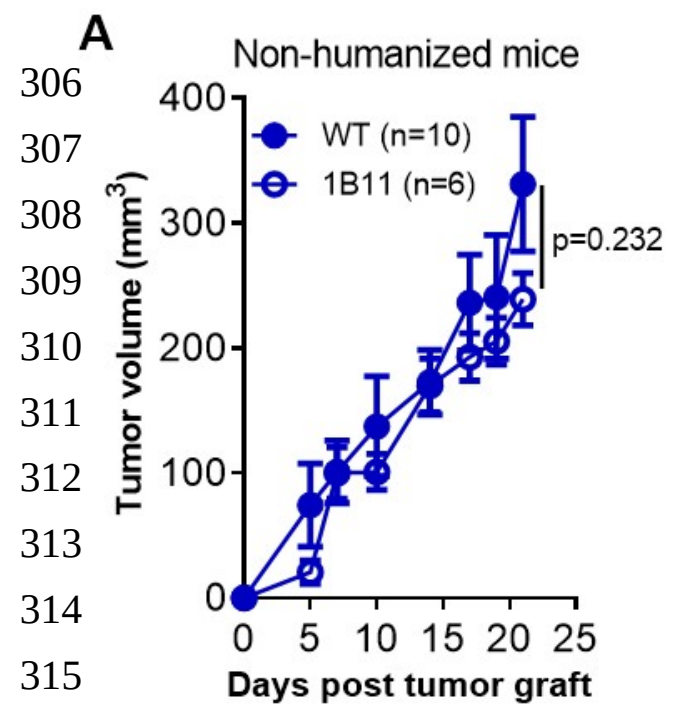

316

317

318

319

320

321

322 linear regression model.

\section{Discussion}

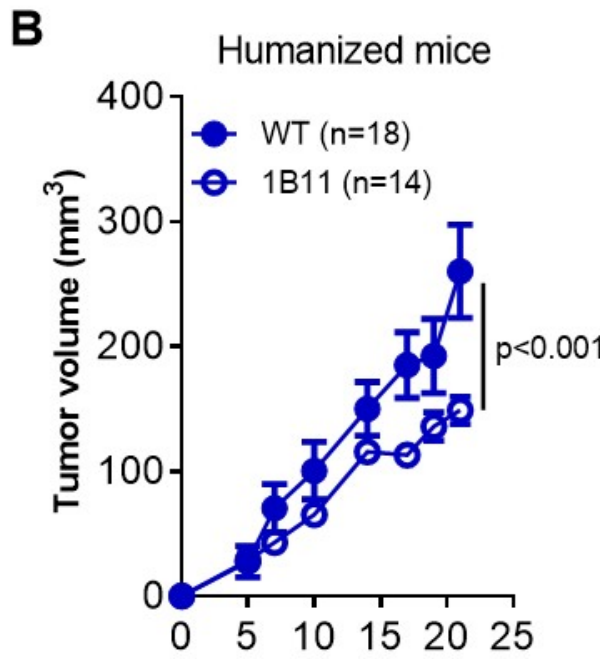

Days post tumor graft
Figure 7: HVEM is an immune checkpoint during anti-tumor $T$ cell immune response in humanized mice. Growth of the indicated PC3 cell lines (WT or 1B11) in nonhumanized (A) or PBMChumanized mice (B). Curves are the mean tumor volume $( \pm$ SEM) in the indicated number of mice. Data are cumulative of at least two experiments. The p value on the graphs indicate the probability that the slopes are equal using a

Here, we report for the first time that HVEM can be targeted by a mAb to improve tumor control by human $\mathrm{T}$ cells in vivo. Moreover, we deciphered the mode of action of the mAb in vivo using complementary technologies. Furthermore, we developed a simple in vivo assay for immune checkpoint discovery and validation. To our knowledge, this is the first report that combine CRISPR/Cas9-mediated deletion of putative checkpoints in the tumor with assessment of tumor growth in humanized mice. One limitation of the assay is that PBMC-humanized mice are mostly reconstituted with $\mathrm{T}$ cells, as shown herein, limiting the usefulness of the assay to T cell-specific immune checkpoints. Nevertheless, we believe that this simple in vivo assay will be of great help to investigate other candidates in more advanced models of humanized mice. 
328 We show that the HVEM/BTLA checkpoint could be exploited for therapy in humanized mice using a mAb

329 to human HVEM. We found that HVEM expression by the tumor was necessary and sufficient to elicit tumor

330 control by the mAb, since the mAb had no effect on HVEM-negative cell lines and no agonist activity on

331 human T cells. Park et al. showed in a syngeneic mouse model that transfecting an agonist scFv anti-HVEM

332 in tumor cells resulted in increased T-cell proliferation, as well as improved IFN- $\gamma$ and IL-2 production and

333 better tumor control [20]. Aside the species differences, the discrepancy with our results could be explained

334 by the fact that T-cells are strongly activated in huPBMC mice [29]. The down regulation of HVEM

335 expression upon activation [30] may have limited the binding of the anti-HVEM antibody on T-cells in our

336 model. Thus, it remains possible that the mAb would behave differently in humans. On the other hand,

337 BTLA is up regulated upon T-cell activation [31], increasing the susceptibility of T-cells to inhibition by

338 HVEM $^{+}$tumor cells [12,14,16,32]. We observed quite the opposite in the tumor micro environment

339 following treatment, with an increase in HVEM and a reduction of BTLA gene expression, with a

340 concomitant increase in LTA and LIGHT, two other ligands for HVEM. It is important to note that the

341 binding sites of LIGHT and BTLA differ on HVEM [33]. So, the anti-HVEM mAb might have limited

342 inhibition of activated T-cells through blockade of HVEM binding with BTLA but not with the other ligands

343 that are T-cell activators. An alternative possibility would be that LIGHT and LTA in their soluble forms

344 inhibit the interaction of HVEM with BTLA [34]. As of today, reciprocal regulation of HVEM and BTLA

345 has not been reported but our observation is reminiscent of earlier findings showing reciprocal regulation of

346 HVEM by LIGHT [30].

347 Previous studies in mice also showed that inhibiting HVEM expression on the tumor or its interaction with

348 its ligands has a positive effect on T cells. Injection of a plasmid encoding a soluble form of BTLA (to

349 compete with endogenous BTLA for HVEM) was associated with an increase in inflammatory cytokines

350 production by TIL and a decrease in anti-inflammatory cytokines at the RNA level [21]. In the same line,

351 vaccination to a tumor-associated antigen was more efficient if HVEM interactions with its ligands were

352 blocked by HSV-1 gD, allowing regression of large tumor mass [35]. Moreover, silencing HVEM expression

353 in the tumor with siRNA was also associated with an increase in CD8 T cells and inflammatory cytokine

354 production in a murine colon carcinoma model [14]. In addition, use of siRNA to HVEM on ovarian cancer

355 in vitro promoted T-cells proliferation and TNF- $\alpha$ and IFN- $\gamma$ production [36]. Numerous results from our 
study also support increased T cell activation in the absence of HVEM/BTLA signaling: TIL from mice treated with anti-HVEM expressed higher levels of JAK-STAT, NFkB and MAPK signaling pathways that are well known inducers of proliferation, differentiation, migration and apoptosis. However, increase in TIL absolute numbers might not be enough to allow tumor rejection. Comparison between TILs from mice treated with the anti-HVEM or isotype control mAb also highlighted decreased expression of many coinhibitory receptors genes (BTLA, TIGIT, LAG3 and TIM3 $[37,38]$ ) or with immunosuppressive functions (CD39 and IL10), suggesting a lower exhaustion status. Overall, we propose that the treatment with the antiHVEM mAb allows better control of tumor growth by increasing the number of cytotoxic CD8 T-cells with a less exhausted phenotype. Our analysis also suggests that this may primarily impacts adhesion and binding in the tumor.

We also identified the impact of myeloid cells of NSG mice during immunotherapy, an overlooked issue when using the model. Our results are in line with published observations relating the crucial role of myeloid cells in tumor control upon immune checkpoints inhibitors treatment in syngeneic mouse models [39-41].

Because of the murine nature of the mAb, binding to murine Fc-receptors present on myeloid cells of NSG might have propelled the therapeutic efficacy of the mAb. In our setting, we used IgG1, that is reported to bind to CD16 (FcgRIII) and CD32 (FcgRIIB), activating and inhibitory receptors, respectively [42].

372 However, NOD background has been associated with a strong decrease in FcgRIIB expression by

373 macrophages [43]. Consequently, activating FcgRIII might be the main receptor involved in FcR-dependent

374 activity of murine myeloid cells in NSG mice. Several possibilities exist to explain tumor killing by myeloid 375 cells, through antibody-dependent cellular phagocytosis (ADCP), local secretion of cytokines or free 376 radicals, expression of FasL and many others [44,45]. We did not see evidence for ADCP on the video microscopy collected during the course of this study, which rather indicated that cell killing was mediated by a cell-contact dependent mechanism, the nature of which remains to be determined. Overall, our data suggest

379 the following model to explain the anti-tumor activity of our anti-HVEM antibody in NSG mice: binding of

380 the mAb on HVEM expressed by the tumor would activate tumor immunogenic cell death by murine 381 myeloid cells, which together with blockade of the HVEM inhibitory network, would limit exhaustion and 382 enhance proliferation and retention/migration of cytotoxic human T-cells. The recent success of ICI for 383 cancer immunotherapy (anti-CTLA-4, anti-PD-1/PD-L1) has confirmed the hypothesis that the immune 
system can control many cancers. In light of the promising results reported herein, anti-HVEM therapy might be combined with ICI and/or chemotherapy to further enhance anti-tumor immunity.

\section{Abbreviations}

HVEM, Herpes Virus Entry Mediator; BTLA, B and T Lymphocyte Attenuator; TIL, Tumor-Infltrating

Leukocytes; TNFSF, Tumor Necrosis Factor Superfamily; NSG, NOD.SCID. $\mathrm{c}^{\text {null; }}$ ADCP, Antibody

Dependent Cellular Phagocytosis; ICI, Immune Checkpoint Inhibitors; RNP, ribonucleoproteins; DEG,

Differentially Expressed Genes; GVHD, Graft-Vs-Host-Disease; IPA, Ingenuity Pathway Analysis; GSEA,

Gene Set Enrichment Analysis

\section{Declarations}

Ethics approval and consent to participate

394 Human peripheral blood mononuclear cells were collected by Etablissement Français du Sang from healthy adult volunteers after informed consent in accordance with the Declaration of Helsinki. Mice were bred in our animal facility under specific pathogen-free conditions in accordance with current European legislation. All protocols were approved by the Ethics Committee for Animal Experimentation Charles Darwin (Ce5/2012/025).

Consent for publication: All authors concur with the submission of the article in its present form

Availability of data and materials: Reagents and datasets are available from the corresponding author on 402 reasonable request.

403 Competing interests: DO declares competing interests as being the co-founder and shareholder of 404 Imcheck Therapeutics, Alderaan Biotechnology and Emergence Therapeutics and has research 405 funds from Imcheck Therapeutics, Alderaan Biotechnology, Cellectis and Emergence Therapeutics. 


\section{Funding}

407 This study was supported by INSERM Transfert, Cancéropôle Île-de-France and Association pour la

408 recherche sur les tumeurs de la prostate (ARTP). The funders play no role in the design of the study and

409 collection, nor in the analysis or interpretation of the data. S.B. was supported by a doctoral fellowship from

410 the French Ministère de l'Education Supérieure et de la Recherche. N.A. is supported by a doctoral

411 fellowship from the Fondation ARC pour la recherche sur le cancer. D.O.'s team was supported by the grant

412 “Equipe FRM DEQ201802339209”. D.O. is Senior Scholar of the Institut Universitaire de France.

\section{Authors' contributions}

414 SB and NA performed the experiments, analyzed the data and contributed to the writing of the manuscript,

415 DO provided essential reagents and edited the manuscript, GM designed the study, analyzed the data and

416 wrote the manuscript.

\section{Acknowledgments}

418 The authors would like to thank Olivier Bregerie and Doriane Foret for taking care of our mice, Dr Pukar KC

419 for technical help, Sylvaine Just-Landi for preparing the $18.10 \mathrm{mAb}$, Dr Armanda Casrouge and Claude

420 Baillou for cell sorting, and Dr Benoit Salomon for critical reading of the manuscript. 


\section{References}

422 1. Hanahan D, Weinberg RA. Hallmarks of cancer: The next generation. Cell. 2011;144:646-74.

423 2. Mittal D, Gubin MM, Schreiber RD, et al. New insights into cancer immunoediting and its three 424 component phases-elimination, equilibrium and escape. Curr Opin Immunol. 2014;27:16-25.

425 3. Hargadon KM, Johnson CE, Williams CJ. Immune checkpoint blockade therapy for cancer: An 426 overview of FDA-approved immune checkpoint inhibitors. Int Immunopharmacol. 2018;62:29-39.

427 4. Havel JJ, Chowell D, Chan TA. The evolving landscape of biomarkers for checkpoint inhibitor 428 immunotherapy. Nat Rev Cancer. 2019;19:133.

429 5. Park Y-J, Kuen D-S, Chung Y. Future prospects of immune checkpoint blockade in cancer: from 430 response prediction to overcoming resistance. Exp Mol Med. 2018;50:109.

431 6. Pasero C, Speiser DE, Derré L, et al. The HVEM network: new directions in targeting novel 432 costimulatory/co-inhibitory molecules for cancer therapy. Curr Opin Pharmacol. 2012;12:478-85.

433 7. Cai G, Anumanthan A, Brown J a, et al. CD160 inhibits activation of human CD4+ T cells 434 through interaction with herpesvirus entry mediator. Nat Immunol. 2008;9:176-85.

435 8. Sedy JR, Gavrieli M, Potter KG, et al. B and T lymphocyte attenuator regulates T cell activation 436 through interaction with herpesvirus entry mediator. Nat Immunol. 2005;6:90-8.

437 9. Cheung TC, Steinberg MW, Oborne LM, et al. Unconventional ligand activation of herpesvirus 438 entry mediator signals cell survival. Proc Natl Acad Sci U S A. 2009;106:6244-9.

439 10. Shaikh RB, Santee S, Granger SW, et al. Constitutive Expression of LIGHT on T Cells Leads to 440 Lymphocyte Activation, Inflammation, and Tissue Destruction. J Immunol. 2001;167:6330-7.

441 11. Harrop JA, McDonnell PC, Brigham-Burke M, et al. Herpesvirus entry mediator ligand 442 (HVEM-L), a novel ligand for HVEM/TR2, stimulates proliferation of T cells and inhibits HT29 443 cell growth. J Biol Chem. 1998;273:27548-56.

444 12. Inoue T, Sho M, Yasuda S, et al. HVEM Expression Contributes to Tumor Progression and 445 Prognosis in Human Colorectal Cancer. Anticancer Res. 2015;35:1361-7.

446 13. Malissen N, Macagno N, Granjeaud S, et al. HVEM has a broader expression than PD-L1 and 447 constitutes a negative prognostic marker and potential treatment target for melanoma.

448 Oncoimmunology. 2019;8.

449 14. Migita K, Sho M, Shimada K, et al. Significant involvement of herpesvirus entry mediator in 450 human esophageal squamous cell carcinoma. Cancer. 2014;120:808-17.

451 15. Lan X, Li S, Gao H, et al. Increased BTLA and HVEM in gastric cancer are associated with 452 progression and poor prognosis. Onco Targets Ther. 2017;10:919-26. 
453 16. Hokuto D, Sho M, Yamato I, et al. Clinical impact of herpesvirus entry mediator expression in 454 human hepatocellular carcinoma. Eur J Cancer. 2015;51:157-65.

455 17. Tsang JYS, Chan K-W, Ni Y-B, et al. Expression and Clinical Significance of Herpes Virus 456 Entry Mediator (HVEM) in Breast Cancer. Ann Surg Oncol. 2017;24:4042-50.

457 18. M'Hidi $\mathrm{H}$, Thibult ML, Chetaille B, et al. High expression of the inhibitory receptor BTLA in T458 follicular helper cells and in B-cell small lymphocytic lymphoma/chronic lymphocytic leukemia. 459 Am J Clin Pathol. 2009;132:589-96.

460 19. Wang Q, Ye Y, Yu H, et al. Immune checkpoint-related serum proteins and genetic variants 461 predict outcomes of localized prostate cancer, a cohort study. Cancer Immunol Immunother. 2020;

20. Park J-J, Anand S, Zhao Y, et al. Expression of anti-HVEM single-chain antibody on tumor cells induces tumor-specific immunity with long-term memory. Cancer Immunol Immunother. 2012;61:203-14.

21. Han L, Wang W, Fang Y, et al. Soluble B and T Lymphocyte Attenuator Possesses Antitumor Effects and Facilitates Heat Shock Protein 70 Vaccine-Triggered Antitumor Immunity against a Murine TC-1 Cervical Cancer Model In Vivo. J Immunol. 2009;183:7842-50.

22. Shultz LD, Schweitzer PA, Christianson SW, et al. Multiple defects in innate and adaptive immunologic function in NOD/LtSz-scid mice. J Immunol. 1995;154:180-91.

23. Schmitz JE, Simon MA, Kuroda MJ, et al. A nonhuman primate model for the selective elimination of CD8+ lymphocytes using a mouse-human chimeric monoclonal antibody. Am J Pathol. 1999/06/11. 1999;154:1923-32.

24. Petit NY, Lambert-Niclot S, Marcelin A-G, et al. HIV Replication Is Not Controlled by CD8+ T Cells during the Acute Phase of the Infection in Humanized Mice. PLoS One. 2015;10:e0138420.

25. Gertner-Dardenne J, Fauriat C, Orlanducci F, et al. The co-receptor BTLA negatively regulates human Vg9Vd2 T-cell proliferation: A potential way of immune escape for lymphoma cells. Blood. 2013;122:922-31.

26. Zhou Y, Zhou B, Pache L, et al. Metascape provides a biologist-oriented resource for the analysis of systems-level datasets. Nat Commun. 2019;10.

27. Subramanian A, Kuehn H, Gould J, et al. GSEA-P: A desktop application for gene set enrichment analysis. Bioinformatics. 2007;23:3251-3.

28. Pasero C, Barbarat B, Just-Landi S, et al. A role for HVEM, but not lymphotoxin- $\beta$ receptor, in LIGHT-induced tumor cell death and chemokine production. Eur J Immunol. 2009;39:2502-14.

29. Ali N, Flutter B, Rodriguez RS, et al. Xenogeneic Graft-versus-Host-Disease in NOD-scid IL2Rcnull Mice Display a T-Effector Memory Phenotype. PLoS One. 2012;7:10. 
31. Murphy KM, Nelson CA, Šedý JR. Balancing co-stimulation and inhibition with BTLA and HVEM. Nat Rev Immunol. 2006;6:671-81.

32. Sasaki Y, Hokuto D, Inoue T, et al. Significance of Herpesvirus Entry Mediator Expression in Human Colorectal Liver Metastasis. Ann Surg Oncol. 2019;26:3982-9.

33. Compaan DM, Gonzalez LC, Tom I, et al. Attenuating lymphocyte activity: The crystal structure of the BTLA-HVEM complex. J Biol Chem. 2005;280:39553-61.

34. Steinberg MW, Cheung TC, Ware CF. The signaling networks of the herpesvirus entry mediator (TNFRSF14) in immune regulation. Immunol Rev. 2011;244:169-87.

35. Lasaro MO, Sazanovich M, Giles-Davis W, et al. Active Immunotherapy Combined With Blockade of a Coinhibitory Pathway Achieves Regression of Large Tumor Masses in Cancer-prone Mice. Mol Ther. 2011;19:1727-36.

36. Zhang T, Ye L, Han L, et al. Knockdown of HVEM, a Lymphocyte Regulator Gene, in Ovarian Cancer Cells Increases Sensitivity to Activated T Cells. Oncol Res Featur Preclin Clin Cancer Ther. 2016;24:189-96.

37. Anderson AC, Joller N, Kuchroo VK. Lag-3, Tim-3, and TIGIT: Co-inhibitory Receptors with Specialized Functions in Immune Regulation. Immunity. 2016;44:989-1004.

38. De Sousa Linhares A, Leitner J, Grabmeier-Pfistershammer K, et al. Not All Immune Checkpoints Are Created Equal. Front Immunol. 2018;9.

39. Dhupkar P, Gordon N, Stewart J, et al. Anti-PD-1 therapy redirects macrophages from an M2 to an M1 phenotype inducing regression of OS lung metastases. Cancer Med. 2018;7:2654-64.

40. Gordon SR, Maute RL, Dulken BW, et al. PD-1 expression by tumour-associated macrophages inhibits phagocytosis and tumour immunity. Nature. 2017;545:495-9.

41. Gubin MM, Esaulova E, Ward JP, et al. High-Dimensional Analysis Delineates Myeloid and Lymphoid Compartment Remodeling during Successful Immune-Checkpoint Cancer Therapy. Cell. 2018;175:1014-1030.e19.

42. Bruhns P. Properties of mouse and human IgG receptors and their contribution to disease models. Blood. 2012;119:5640-9.

43. Luan JJ, Monteiro RC, Sautès C, et al. Defective Fc gamma RII gene expression in macrophages of NOD mice: genetic linkage with up-regulation of IgG1 and IgG2b in serum. $J$ Immunol. 1996;157:4707-16.

44. Furness AJS, Vargas FA, Peggs KS, et al. Impact of tumour microenvironment and Fc receptors on the activity of immunomodulatory antibodies. Trends Immunol. 2014;35:290-8.

45. Gül N, Van Egmond M. Antibody-dependent phagocytosis of tumor cells by Macrophages: A Potent effector mechanism of monoclonal antibody therapy of cancer. Cancer Res. 2015;75:500813. 


\section{Supplementary Figures}

A

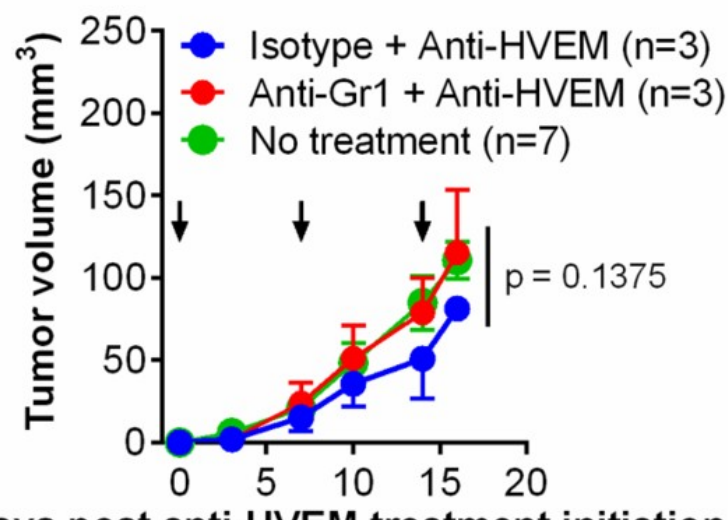

B

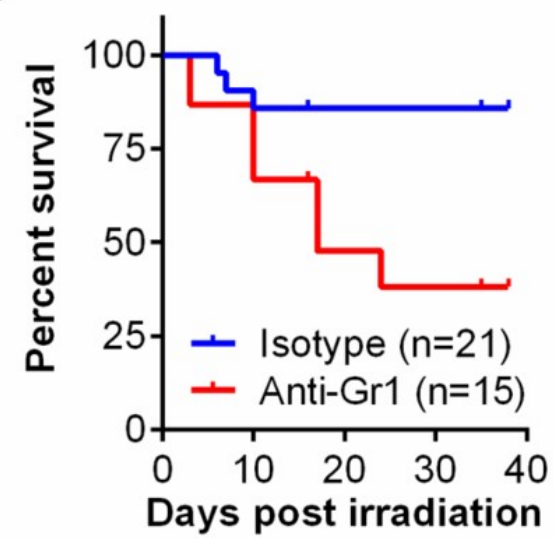

Figure S1: Depletion of Gr1+ cells reverted the effect of the anti-HVEM mAb in non humanized NSG mice. (A) Tumor growth of wild-type PC3 grafted in NSG mice treated with anti-HVEM (arrows) and anti-

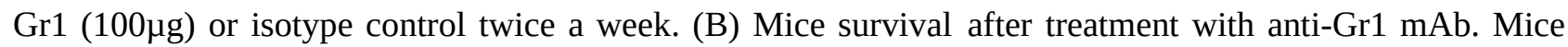
were treated with various doses of two different batches of anti-Gr1. Data are cumulative from 3 independent experiments.
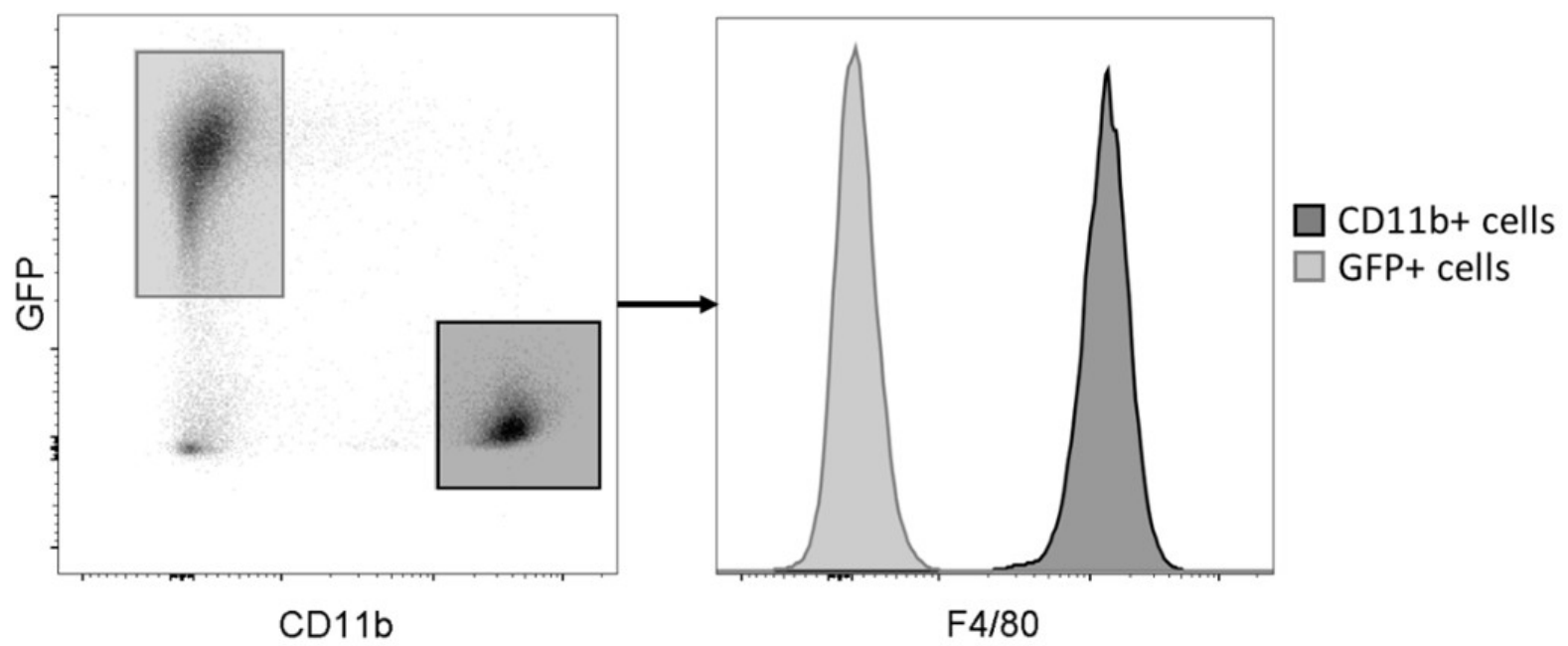

531 Figure S2: Co-cultures of GFP-PC3 cell line and cells from peritoneal lavage of NSG mice. Cells were

532 stained with anti-CD11b and F4/80-specific mAb and analyzed by flow cytometry at the initiation of the co533 culture. 


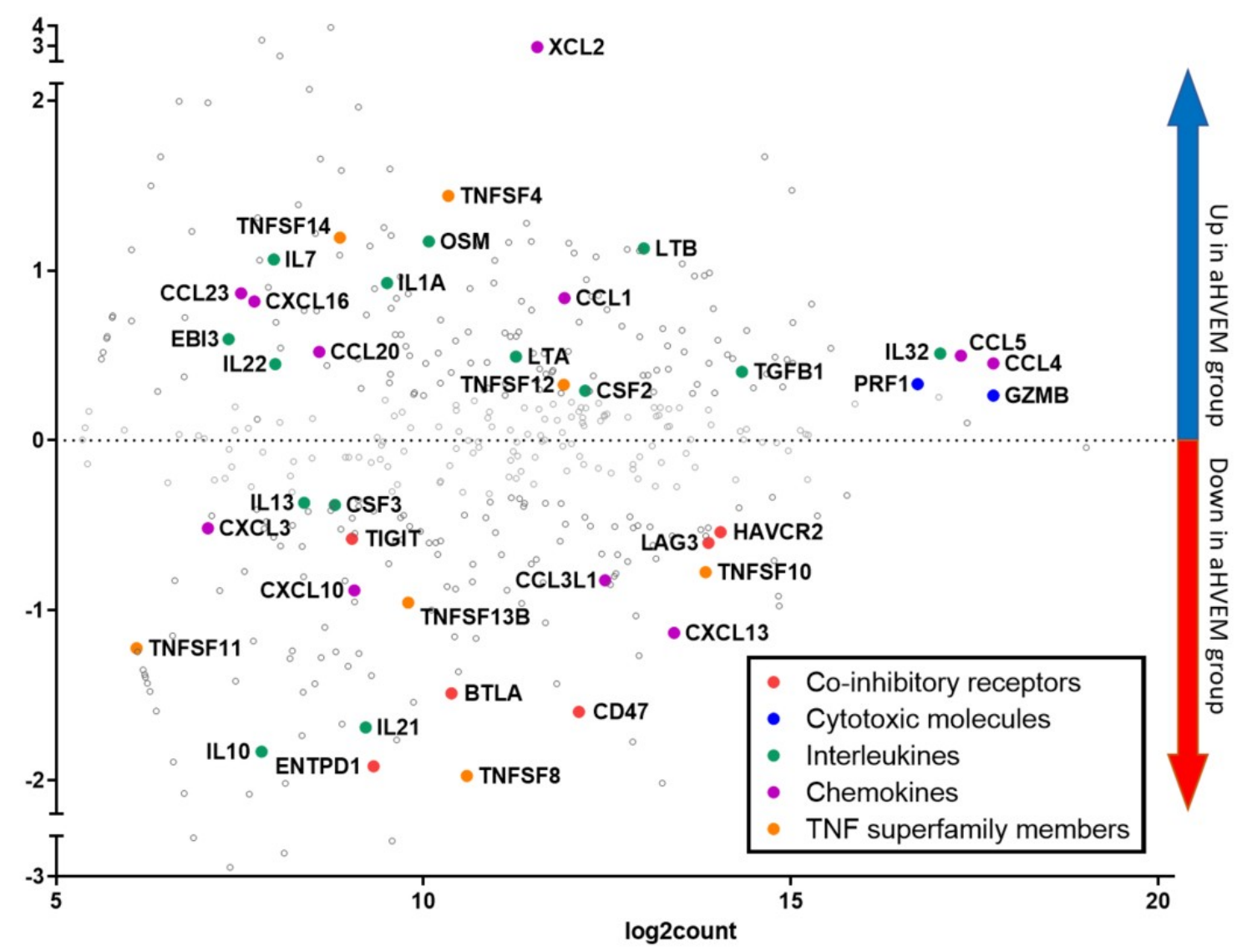

534 Figure S3: MA-plot comparing gene expression between TIL from aHVEM and isotype treated mice.

535 Represented are the fold-change in the expression of a given gene between anti-HVEM- or isotype-treated

536 mice (log2FC, y axis) vs the mean absolute count after normalization (log2count). Some notable genes are

537 highlighted according to their biological functions by the indicated color code. 
A

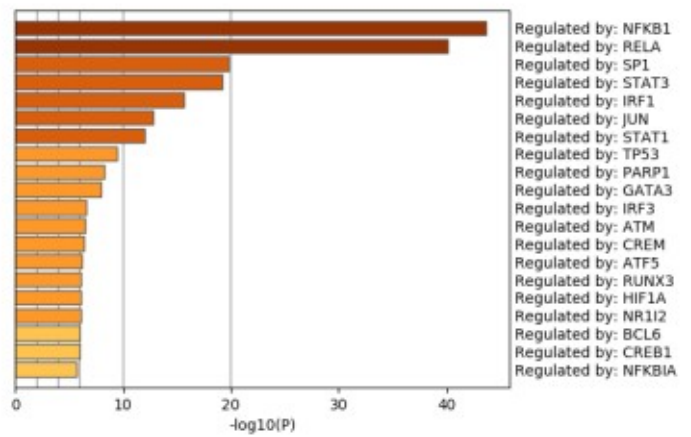

B

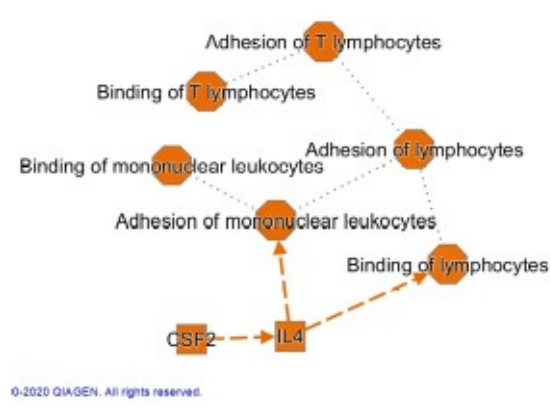

538 Figure S4: Enrichment analysis of DEG in TIL of anti-HVEM-treated mice (A) Putative regulators of

539 DEG were determined with Metascape and the TRRUST database (B) Representation of the most significant

540 biological features from the DEG of anti-HVEM treated mice. The network was generated using the

541 Graphical Summary algorithm of the Ingenuity Pathway Analysis software (Qiagen).

\section{Video S1: NSG macrophages are able to kill PC3 cell in presence of anti-HVEM by a cell-contact}

543 dependent mechanism. GFP-expressing PC3 cells were incubating with NSG peritoneal macrophages and

544 anti-HVEM or its isotype. Co-culture was followed by video microscopy overnight. 